\title{
Gelatin nanoparticle-mediated intranasal delivery of substance $P$ protects against 6-hydroxydopamine- induced apoptosis: an in vitro and in vivo study
}

This article was published in the following Dove Press journal:

Drug Design, Development and Therapy

7 April 2015

Number of times this article has been viewed

\author{
Cui-Tao Lu' ${ }^{1,2}$ \\ Rong-Rong Jin ${ }^{2}$ \\ Yi-Na Jiang ${ }^{2}$ \\ Qian Lin ${ }^{2}$ \\ Wen-Ze Yu ${ }^{2}$ \\ Kai-Li Mao ${ }^{2}$ \\ Fu-Rong Tian ${ }^{2}$ \\ Ya-Ping Zhaol,* \\ Ying-Zheng Zhao $2, *$ \\ 'The Second Affiliated Hospital, \\ Wenzhou Medical University, ${ }^{2}$ School \\ of Pharmaceutical Sciences, Wenzhou \\ Medical University, Wenzhou, \\ People's Republic of China \\ *These authors contributed equally \\ to this work
}

Background: The aim of this study was to investigate the protective role of intranasally administered substance P-loaded gelatin nanoparticles (SP-GNPs) against 6-hydroxydopamine (6-OHDA)-induced apoptosis in vitro and in vivo, and to provide a new strategy for treating brain pathology, such as Parkinson's disease.

Methods: SP-GNPs were prepared by a water-in-water emulsion method, and their stability, encapsulating efficiency, and loading capacity were evaluated. PC-12 cells were used to examine the enhancement of growth and inhibition of apoptosis by SP-GNPs in vitro using MTT assays. In the in vivo study, hemiparkinsonian rats were created by intracerebroventricular injection of 6-OHDA. The rats then received intranasal SP-GNPs daily for 2 weeks. Functional improvement was assessed by quantifying rotational behavior, and the degree of apoptosis was assessed by immunohistochemical staining for caspase-3 in the substantia nigra region.

Results: PC-12 cells with 6-OHDA-induced disease treated with SP-GNPs showed higher cell viability than their untreated counterparts, and cell viability increased as the concentration of substance P (SP) increased, indicating that SP could enhance cell growth and inhibit the cell apoptosis induced by 6-OHDA. Rats with 6-OHDA-induced hemiparkinsonism treated with SP-GNPs made fewer rotations and showed less staining for caspase-3 than their counterparts not treated with SP, indicating that SP protects rats with 6-OHDA-induced hemiparkinsonism from apoptosis and therefore demonstrates their functional improvement.

Conclusion: Intranasal delivery of SP-GNPs protects against 6-OHDA-induced apoptosis both in vitro and in vivo.

Keywords: gelatin nanoparticles, intranasal delivery, substance P, 6-hydroxydopamine, apoptosis, Parkinson's disease

\section{Introduction}

A hydroxyl derivative of catecholamine, 6-hydroxydopamine (6-OHDA) is a neurotoxicant that activates apoptotic and proapoptotic factors, eg, caspase proteins, as well as transduction of Bax factor, leading to apoptosis and degeneration of dopamine neurons. ${ }^{1-3}$ Studies have shown that 6-OHDA can induce apoptosis in PC-12 (adrenal pheochromocytoma) cells by activating apoptotic factors. ${ }^{2}$ Also, rats intracerebroventricularly injected with 6-OHDA show apoptosis, degeneration, and death of dopaminergic neurons in the substantia nigra. The apoptosis occurs mainly due to a caspase family member-mediated protease cascade, and caspase- 3 has a vital role in this process. If large numbers of dopaminergic neurons undergo apoptosis, the result is irreversible degenerative brain disease, ie, Parkinson's disease (PD), for which there is still no effective therapy. ${ }^{4}$

Substance P (SP), a member of the tachykinin peptide family, is involved in the regulation of many biological processes in the central and peripheral nervous 
systems (Figure 1). ${ }^{5}$ SP-containing neurons are widely distributed throughout the central and peripheral nervous systems, especially in the substantia nigra region. ${ }^{6}$ Most SP receptors are located within dopaminergic and cholinergic neurons in the basal ganglia, suggesting that SP may have a physiologically regulating effect on these neurons. ${ }^{7}$ Therefore, $\mathrm{SP}$ and its receptor may have a therapeutic use in $\mathrm{PD}$, which is characterized by impaired dopaminergic transmission.

It has been reported that SP and dopamine are regulated via a positive feedback mechanism whereby binding of SP to its tachykinin neurokinin-1 receptor on dopamine neurons causes striatal release of dopamine, and by binding to its D1 receptor on striatal projection neurons, dopamine potentiates the release of SP within the substantia nigra. ${ }^{8}$ Previous research has shown that expression of SP is significantly decreased in the basal nuclei in both hemiparkinsonian rats and $\mathrm{PD}$ patients, indicating probable involvement of SP in regulating the pathogenesis of PD. ${ }^{7}$ In vitro experiments have demonstrated that SP can reduce antiFas-induced apoptosis in human tenocytes via regulation of neurokinin-1-specific and Akt-specific pathways. ${ }^{9}$ An vivo study suggested that SP can reduce apoptotic cell death by modulating the immune response in the early stages after spinal cord injury. ${ }^{10}$

Intracerebroventricular administration of SP to rats with 6-OHDA-induced disease can increase the dopamine content in the brain and help to restore the dopamine deficit, with the positive effects seen being more prominent in the nigrostriatal system than in the mesocorticolimbic dopaminergic system. ${ }^{11}$ Further, hemiparkinsonian rats pretreated with SP fragments $^{12}$ or an SP receptor antagonist ${ }^{13}$ show increased levels of dopamine and its metabolites in the corpus striatum, as well as clear functional recovery. However, the current research focuses on the pharmacological effects of SP given by invasive intracerebroventricular injection, which can result in a high local concentration of SP in the brain, and it has been confirmed that a high level of SP in the brain can induce serious neuroinflammation and further aggravate illness, ${ }^{8,14,15}$ so intracerebroventricular injection is not a safe or practical strategy for PD patients who need continuous treatment.

Intranasal administration has been reported to be an efficient and noninvasive way to delivery biologics directly into the brain. ${ }^{16}$ Gelatin nanoparticles (GNPs) are a type of gelatin-cored nanostructured lipid carrier prepared by a water-in-water emulsion method and have good stability and strong penetrating ability, encapsulating efficiency, and loading capacity, as well as bioactivity. ${ }^{17}$ It has been reported that GNPs are a suitable carrier for targeted delivery, making it possible to delivery therapeutics to a focal zone effectively without compromising drug stability or concentration. ${ }^{18,19}$ Theoretically, a novel strategy combining GNP-loaded therapeutics and the nasal olfactory pathway might maximize the potential efficacy of SP in the treatment of PD.

In the present study, we investigated whether intranasally administered SP-GNPs could maximize the ability of SP to protect against 6-OHDA-induced apoptosis in vitro and in vivo. SP-GNPs were prepared by a water-in-water emulsion method and were found to have good stability, encapsulating efficiency, and loading capacity. The protective effect of SP-GNPs on PC-12 cells with 6-OHDA-induced disease was assessed by MTT [3-(4,5-dimethylthiazol-2-yl)2,5-diphenyltetrazolium bromide] assay, and the inhibition of apoptosis and neuroprotective effect of SP-GNPs in rats<smiles>CSCCC(NC(=O)[C@H](CC(C)C)NC(=O)CNC(=O)[C@H](Cc1ccccc1)NC(=O)[C@H](Cc1ccccc1)NC(=O)[C@H](CCC(N)=O)NC(=O)[C@H](CCC(N)=O)NC(=O)[C@@H]1CCCN1C(=O)[C@H](CCCCN)NC(=O)[C@@H]1CCCN1C(=O)[C@@H](N)CCCNC(=N)N)C(N)=O</smiles>

H-Arg-Pro-Lys-Pro-GIn-GIn-Phe-Phe-Gly-Leu-Met-NH ${ }_{2}$

Figure I Molecular structure of substance P. 
with 6-OHDA-induced hemiparkinsonism were evaluated by behavioral assessment and immunohistochemical staining for caspase-3.

\section{Materials and methods Materials and animals}

All the materials and equipment used in this study were commercially available. SP was purchased from GL Biochem Ltd (Shanghai, People's Republic of China). The protocols and procedures were approved by the local animal experimentation ethics committee. Male Sprague-Dawley rats weighing around 300-320 g were provided by the Laboratory Animal Services Centre at our university. Two to three animals were housed per stainless steel cage on a 12-hour light/12-hour dark cycle in an air-conditioned room at $22^{\circ} \mathrm{C}$, and checked daily by the animal care staff. Standard commercial rat chow (Prolab RMH 2500, PMI Nutrition International LLC, Brentwood, MO, USA) and water were available ad libitum.

\section{Preparation and characterization of SP-GNPs and blank GNPs}

SP-GNPs and blank GNPs were prepared using waterin-water emulsion and freeze-drying techniques. ${ }^{20,21}$ Briefly, a high concentration of SP was dissolved in $1 \mathrm{~mL}$ of $20 \%$ w/v Poloxamer 188-grafted heparin copolymer solution. This solution was added to $2 \mathrm{~mL}$ of $2.0 \% \mathrm{w} / \mathrm{v}$ gelatin solution to produce a homogeneous mixture. Under sonication $\left(110 \mathrm{~W}, 15^{\circ} \mathrm{C}\right)$ using a probe sonicator, D,L-glyceraldehyde was injected into the mixed solution until its final concentration reached $0.1 \% \mathrm{w} / \mathrm{v}$ to initiate the cross-linking reaction. The mixture was bathed at $5^{\circ} \mathrm{C}$ under magnetic stirring at 2,500 rpm for 5 hours to form a suspension of SP and GNPs. The suspension was lyophilized to obtain a powder containing SP and polymeric GNPs. Next, the lyophilized powder was dispersed in a solution containing soy phosphatidylcholine, trehalose, and cholesterol. By sonication $(90 \mathrm{~W}$, 20 seconds) at $25^{\circ} \mathrm{C}$, the suspension was then lyophilized to obtain a powder containing SP-GNPs, which were reconstituted in double-distilled water to form a $2 \mathrm{mg} / \mathrm{mL}$ SP-GNP suspension for administration. Blank GNPs (using gelatin solution instead of SP gelatin solution during preparation) was also prepared for the subsequent experiment.

The morphologies of the SP-GNPs and blank GNPs were determined using a scanning electron microscope (X-650, Hitachi, Tokyo, Japan). The particle size and zeta potential were determined by dynamic light scattering using a Nicomp ${ }^{\text {TM }} 380$ ZLS zeta potential/particle sizer (PSS Nicomp, Santa Barbara, CA, USA).
To determine the encapsulating efficiency of the SPGNPs and blank GNPs, approximately $1.5 \mathrm{~mL}$ of the SP-GNP dispersion were placed in a microtube and centrifuged at $10,000 \mathrm{~g}$ for 40 minutes. The supernatant was then collected and diluted for determination of SP content using an enzyme-linked immunosorbent assay kit; this experiment was performed in triplicate. Drug encapsulation efficiency $(\%)=$ (total amount of drug - amount of drug in supernatant)/total amount of drugs added initially $\times 100 \%$.

\section{Experiment in vitro Cell culture}

Male rat PC-12 cells were used for the in vitro study. The PC-12 cells were cultured at $37^{\circ} \mathrm{C}$ in high-glucose Dulbecco's Modified Eagle's Medium with 10\% fetal bovine serum and $1 \%$ penicillin-streptomycin in a humidified incubator containing $5 \% \mathrm{CO}_{2}$. Cells in the logarithmic growth phase were harvested with trypsin for further experiments.

\section{MTT assay}

The ability of SP-GNPs to impede the growth of PC-12 cells with 6-OHDA-induced disease was confirmed by MTT assay (run in triplicate). PC-12 cells were cultured in a 96-well plate for 24 hours at a density of 5,000 cells per well. With blank PC-12 cells as the control, $100 \mu \mathrm{M}$ of 6-OHDA was added to the cells for 24 hours to induce cell apoptosis, after which blank GNPs and different concentrations of SP-GNPs were incubated for another 24 hours. Next, $10 \mu \mathrm{L}$ of MTT $5 \mathrm{mg} / \mathrm{mL}$ were added to each well and incubated for 4 hours; $100 \mu \mathrm{L}$ of formazan solution was then added to each well, followed by incubation for a further 4 hours to dissolve the crystals that developed in each well. The plate was then put into a microplate reader to measure the optical density at $526 \mathrm{~nm}$ and quantify the extent of cell viability. The higher the amount of cell viability in each well, the less the degree of apoptosis.

\section{Experiment in vivo}

\section{Rat model of hemiparkinsonism}

The rats were anesthetized with pentobarbital sodium $60 \mathrm{mg} / \mathrm{kg}$ and then injected with $12 \mu \mathrm{L}$ of 6-OHDA solution into the right striatum (or vehicle for sham animals) using stereotaxic apparatus (Figure 2). ${ }^{22,23}$ Gentamicin was then given to prevent infection.

Four weeks after injection of the 6-OHDA solution, rodent behavior was evaluated by counting the number of apomorphine-induced rotations to determine if the rat model 
of hemiparkinsonism had been successfully created. The rats were injected with apomorphine $0.5 \mathrm{mg} / \mathrm{kg}$ subcutaneously, and both contralateral and ipsilateral full-body rotations were recorded in the following 30 minutes. At least seven full-body contralateral rotations per minute were considered to indicate a successful hemiparkinsonian (PD) model, and these rats were used in the following experiment.

\section{Effect of SP-GNPs in hemiparkinsonian rats}

The day after the behavior evaluation, the sham rats and PD rats were randomized into five groups ( $n=8$ per group) and started on daily treatment for 2 weeks. Group 1 comprised sham rats receiving intranasal phosphate-buffered saline and group 2 comprised PD rats receiving intranasal blank GNPs. Groups 3, 4, and 5 comprised PD rats receiving intranasal SP-GNPs at different concentrations (Table 1).

Two hours after the end of 2 weeks of daily treatment, the experimental rats were injected subcutaneously with apomorphine $0.5 \mathrm{mg} / \mathrm{kg}$ to evaluate the extent of their neurorecovery. All contralateral and ipsilateral full-body rotations were recorded during the 30 minutes following injection of apomorphine. The fewer the number of rotations in the hemiparkinsonian rats, the better the neurorecovery was deemed to be.

All rats were euthanized at this point, and their brains were collected for coronal sectioning across the substantia nigra region. The brain tissues were then embedded in paraffin and sectioned for immunohistochemical staining.

\section{Immunohistochemical staining}

As one of the endpoint shear enzymes in apoptosis, caspase-3 plays a critical role in the apoptotic cascade. ${ }^{24}$ Immunohistochemical staining with anti-caspase- 3 antibody was used to evaluate levels of apoptosis in the substantia nigra region in hemiparkinsonian rats treated or not treated with SP. ImagePro Plus version 6.0 was used to quantify the number of cells, the areas stained, and the degree of staining. The better the

Table I Rat groups designed for 2 weeks of daily experimental treatment ( $n=8$ per group)

\begin{tabular}{llll}
\hline Group & $\begin{array}{l}\text { Rat } \\
\text { model }\end{array}$ & $\begin{array}{l}\text { Intranasal } \\
\text { treatment }\end{array}$ & $\begin{array}{l}\text { SP dosage } \\
(\mu \text { g/day })\end{array}$ \\
\hline I & Sham & PBS & \\
2 & PD & Blank GNPs & \\
3 & PD & SP-GNPs & 50 \\
4 & PD & SP-GNPs & 75 \\
5 & PD & SP-GNPs & 100 \\
\hline
\end{tabular}

Abbreviations: PD, Parkinson's disease; PBS, phosphate-buffered saline; SP, substance P; GNPs, gelatin nanoparticles. protective effect against 6-OHDA-induced apoptosis, the lower rates of caspase-3 staining and apoptosis in brain sections from the PD rats.

\section{Statistical analysis}

Statistically significant differences across multiple groups were determined using one-way analysis of variance with the Newman-Keuls post hoc test. Statistically significant differences between individual groups was determined using the Mann-Whitney $U$-test. All testing was done using Statistical Package for the Social Sciences version 19 software (SPSS Inc, Chicago, IL, USA). A difference was considered to be statistically significant at $P<0.05$.

\section{Results}

Physicochemical properties and bioactivity of SP-GNPs and blank GNPs

Scanning electron micrographs showed that the SP-GNPs and blank GNPs were uniform in shape and size (Figure 3). Characterization for the SP-GNPs and blank GNPs is shown in Table 2. Dynamic light scattering showed the average particle size of the blank GNPs to be $136 \pm 1.32 \mathrm{~nm}$.

The polydispersity index (PDI) indicates the distribution of particle size. Low PDI values were observed for the SP-GNPs and the blank GNPs (Table 2), indicating that both were monodispersed stable systems. After loading with SP, the mean diameters of the nanoparticles and liposomes increased, but were still below $200 \mathrm{~nm}$ (Table 2).

The zeta potential is an important indicator of the physical stability of nanoparticles. Nanoparticles with a high absolute zeta potential value are electrically stable while those with a low absolute zeta potential value tend to be less electrically stable. As shown in Table 2, both the SP-GNPs and the blank GNPs had a strong negative surface charge, indicating that coating with phospholipids makes these nanoparticles more stable.

The encapsulation efficiency and loading capacity of the SP-GNPs were $93.3 \pm 1.4 \%$ and $5.2 \pm 0.02 \%$, respectively (Table 2).

\section{MTT assays}

Table 3 shows the ability of different concentrations of SP-GNPs to limit the growth of PC-12 cells with 6-OHDAinduced disease. When compared with untreated PC-12 cells with 6-OHDA-induced disease, those treated with blank GNPs showed slightly higher but not significantly different cell viability, whereas their counterparts treated with SP-GNPs did demonstrate significantly higher cell viability 

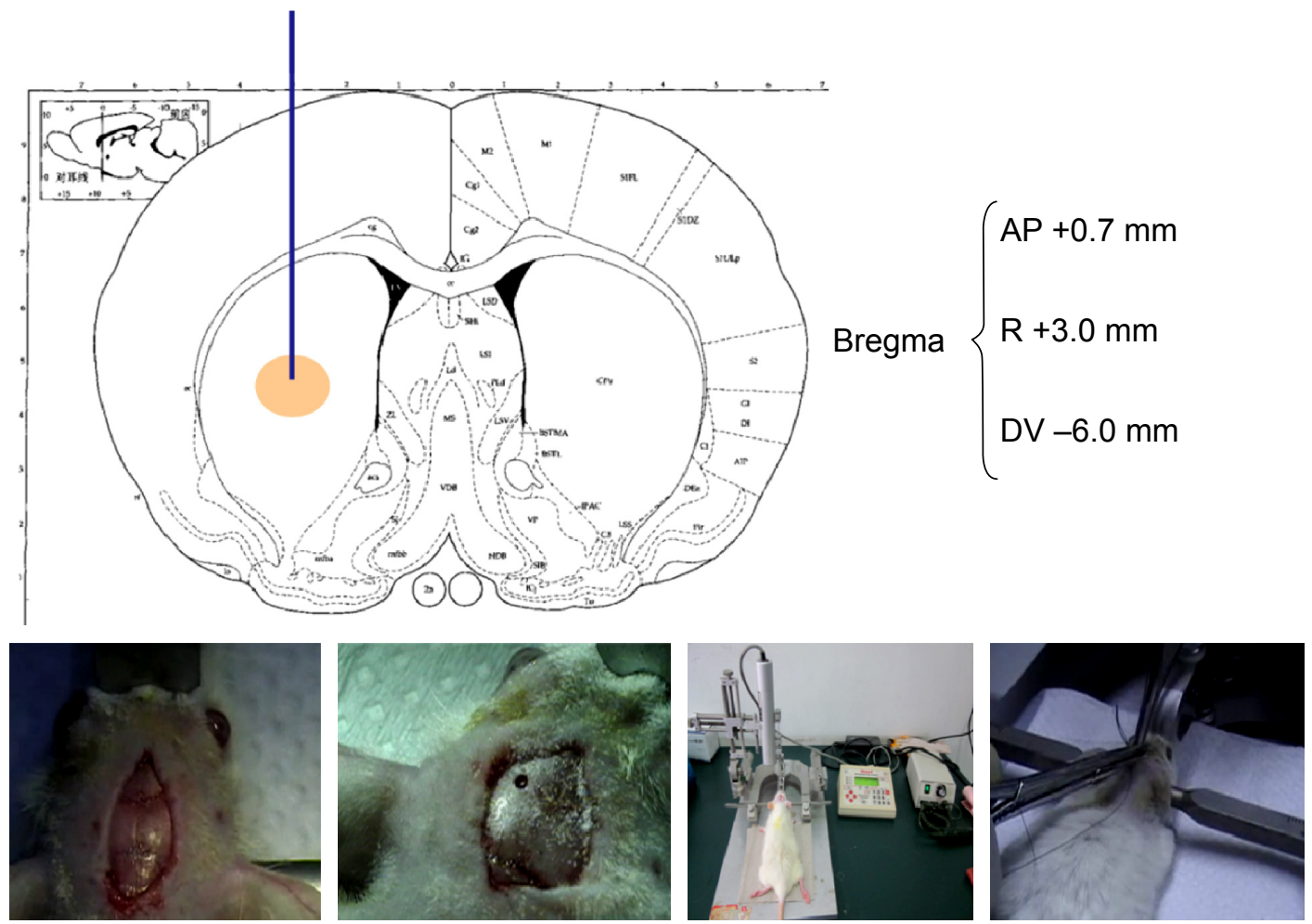

Figure 2 Rat model of hemiparkinsonism.

Note: The anesthetized SD rats were placed on stereotaxic apparatus and then injected with I2 $\mu \mathrm{L} 6-\mathrm{OHDA}$ solution (or vehicle for sham animals) in the right-side striatum. Abbreviations: AP, distance after the fontanelle; $R$, movement to the right side; $D V$, depth value.

$(P<0.05)$, indicating that SP-GNPs can decrease the extent of apoptosis caused by 6-OHDA and enhance cell growth. In the meantime, cell viability increased as the concentration of SP increased, suggesting that within a certain range of concentrations, the degree of inhibition of apoptosis achieved by SP is concentration-dependent.

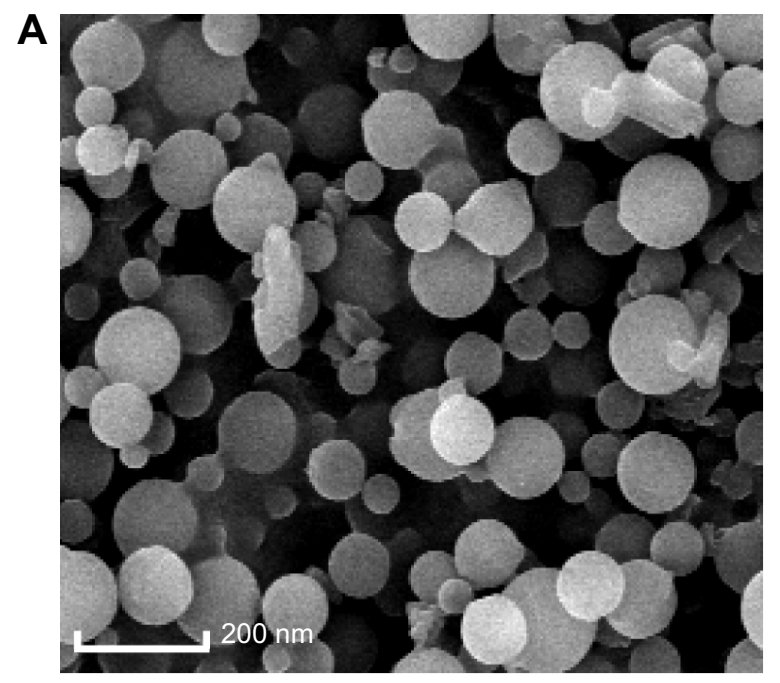

Figure 3 Scanning electron micrographs of blank GNPs (A) and SP-GNPs (B). Abbreviations: SP, substance P; GNPs, gelatin nanoparticles.

\section{Behavioral evaluation of PD rats after 2 weeks of treatment}

The number of apomorphine-induced rotations following 2 weeks of daily treatment with SP-GNPs in each experimental group were consistent with the dopamine levels in the diseased brain. As seen in Table 4, the PD rats that received

B

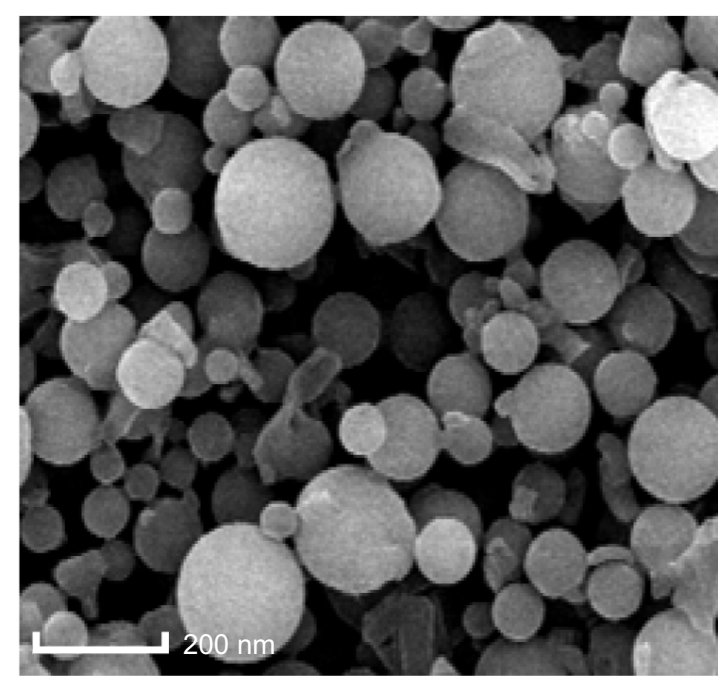


Table 2 Characterization of gelatin nanoparticles and liposomes with or without substance $P(n=3)$

\begin{tabular}{llllll}
\hline Formulation & Particle size $(\mathbf{n m})$ & PDI & Zeta potential $(\mathbf{m V})$ & EE $(\%)$ & Loading capacity $(\%)$ \\
\hline Blank GNPs & $166 \pm 1.32$ & $0.145 \pm 0.021$ & $-37.6 \pm 1.4$ & - & - \\
SP-GNPs & $172 \pm 1.52$ & $0.107 \pm 0.013$ & $-29.6 \pm 1.2$ & $93.3 \pm 1.4$ & $5.2 \pm 0.02$ \\
\hline
\end{tabular}

Abbreviations: SP, substance P; GNPs, gelatin nanoparticles; EE, encapsulation efficiency; PDI, polydispersity index.

intranasal SP-GNP treatment made fewer rotations than those that received blank GNPs, demonstrating that SP could protect dopaminergic neurons against 6-OHDA-induced apoptosis and aid recovery of diseased dopaminergic neurons in rats with PD. Meanwhile, the PD rats that received daily intranasal treatment with $75 \mu \mathrm{g}$ or $100 \mu \mathrm{g}$ of SP showed a significant decrease in the number of apomorphine-induced rotations when compared with hemiparkinsonian rats $(P<0.05)$, while PD rats that received $50 \mu \mathrm{g}$ of SP per day intranasally showed a trend towards improvement $(P>0.05)$, indicating that the extent of the protective effect of SP against 6-OHDA-induced apoptosis has a positive relationship with the concentration of SP.

\section{Immunohistochemical staining of caspase- 3 in the substantia nigra}

As seen in Figure 4, immunohistochemical staining for caspase- 3 in the diseased substantia nigra was limited in the sham group but extensive in the PD group, indicating that caspase- 3 is rarely expressed in normal circumstances but is expressed in large amounts in the presence of PD. Less staining was seen in the SP-GNP groups than in the blank GNP group, suggesting that SP-GNPs can inhibit the expression of caspase-3 and reduce neuronal apoptosis, thus helping the diseased neurons to recover. Further, PD rats receiving $75 \mu \mathrm{g}$ or $100 \mu \mathrm{g}$ of intranasal SP per day showed significantly less caspase-3 staining than PD rats that did not receive SP $(P<0.05)$, while PD rats receiving $50 \mu \mathrm{g}$ of intranasal SP per day showed slightly lower level of caspase-3 staining than PD rats $(P>0.05)$, indicating that the higher the concentration

Table 3 Results of MTT assay on PC- 12 cells

\begin{tabular}{ll}
\hline Group & Cell viability \\
\hline Sham & $1.0000 \pm 0.5397$ \\
6-OHDA disease & $0.3562 \pm 0.3173$ \\
Blank GNPs & $0.4036 \pm 0.3087$ \\
I nM SP-GNPs & $0.4817 \pm 0.243 I^{*}$ \\
I0 nM SP-GNPs & $0.5239 \pm 0.2345^{*}$ \\
I00 nM SP-GNPs & $0.5966 \pm 0.255 I^{* *}$ \\
\hline
\end{tabular}

Notes: $* P<0.05, * * P<0.01$ versus group with 6-OHDA disease.

Abbreviations: SP, substance P; GNPs, gelatin nanoparticles; 6-OHDA, 6-hydroxydopamine; MTT, 3-(4,5-dimethylthiazol-2-yl)-2,5-diphenyltetrazolium bromide; PC-12, adrenal pheochromocytoma. of SP, the better the effect in protecting against 6-OHDAinduced neuronal apoptosis.

\section{Discussion}

6-OHDA is a neurotoxin that activates the apoptotic cascade in the central nervous system, leading to apoptosis and degeneration of dopaminergic neurons, which culminates in cell apoptosis in vitro and PD in vivo. SP, a member of the tachykinin peptide family, has been shown to play an important role in protecting against neurotoxin-induced apoptosis.

Studies show that drugs or particles smaller than $300 \mathrm{~nm}$ can bypass the blood-brain barrier, can be absorbed through the mucous membrane in the nasal olfactory region, and can be delivered into the brain directly through the cribriform plate, beyond which they exert their therapeutic effects in specific regions inside the brain. ${ }^{25-27}$ In earlier studies, nanoparticles were used as intranasal carriers for therapeutics to enable effective treatment of brain disorders, such as cerebral ischemia ${ }^{28}$ and PD. ${ }^{29}$ It is reported that nanoparticles administered intranasally can penetrate the brain through several pathways: the olfactory pathway, in which particles are taken up by the olfactory epithelium and the olfactory bulb; the trigeminal pathway, in which particles are delivered along the trigeminal nerve system; the vascular pathway, in which particles are absorbed into the capillaries underlying the nasal mucosa; and other pathways, such as cerebrospinal fluid and the lymphatic system. ${ }^{30-32}$ However, because of the mucociliary clearance mechanism in the nose, particles cannot be lodged in the nasal cavity for a long period, which

Table 4 Apomorphine-induced rotations after 2 weeks of daily treatment with substance $P$ ( $n=8$ per group)

\begin{tabular}{lllll}
\hline Group & $\begin{array}{l}\text { Rat } \\
\text { model }\end{array}$ & $\begin{array}{l}\text { Intranasal } \\
\text { treatment }\end{array}$ & $\begin{array}{l}\text { SP dosage } \\
(\mu \text { g/day })\end{array}$ & $\begin{array}{l}\text { Rotations } \\
\text { per minute }\end{array}$ \\
\hline I & Sham & PBS & & 0 \\
2 & PD & Blank GNPs & & $7.6 \pm 0.5$ \\
3 & PD & SP-GNPs & 50 & $6.8 \pm 1.1$ \\
4 & PD & SP-GNPs & 75 & $6.5 \pm 1.3^{*}$ \\
5 & PD & SP-GNPs & 100 & $6.3 \pm 1.7^{*}$ \\
\hline
\end{tabular}

Note: $* P<0.05$ versus group 2.

Abbreviations: PD, Parkinson's disease; PBS, phosphate-buffered saline; SP, substance P; GNPs, gelatin nanoparticles. 

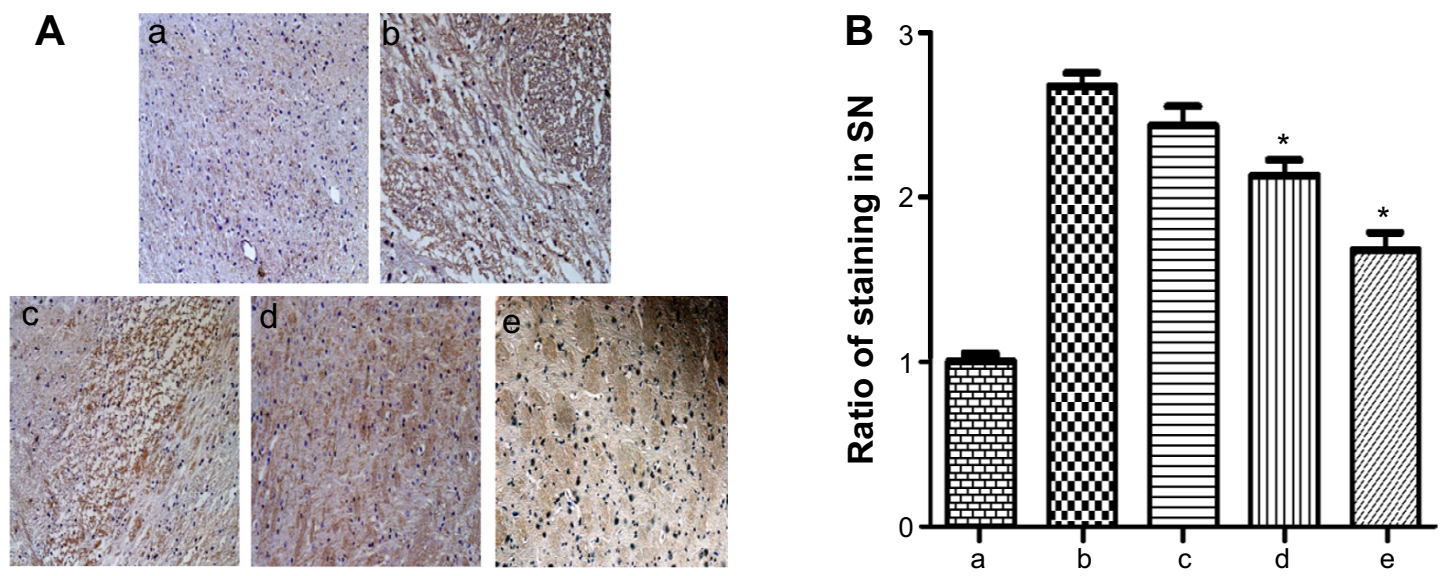

Figure 4 Immunohistochemical staining of caspase-3 in the SN region.

Notes: (A) Results of caspase-3 staining in SN region. (B) Quantification of caspase-3 staining in SN region. (a) Sham group. (b) PD rats intranasally administered blank GNP. (c) PD rats intranasally administered SP-GNP $50 \mu \mathrm{g} /$ day. (d) PD rats intranasally administered SP-GNP $75 \mu \mathrm{g} /$ day. (e) PD rats intranasally administered SP-GNP I00 $\mu \mathrm{g} /$ day. Abbreviations: PD, Parkinson's disease; SN, substantia nigra; SP, substance P; GNP, gelatin nanoparticle.

limits the application of intranasal administered drug-loaded particles. $^{27,33}$

In recent years, gelatin and nonionic surfactants (such as Poloxamer 188) have been used to prepare nanoparticles due to their biocompatibility, biodegradability, low immunogenicity, and amenability for surface modification. ${ }^{34-36}$ Nanoparticles modified with gelatin have a negative charge that can reduce mucociliary clearance, extend the residence time at the site of delivery, and enhance the therapeutic effect when administered intranasally. ${ }^{17-19}$ In a previous study, we found that gelatin nanostructured lipid carrier-mediated intranasal delivery of basic fibroblast growth factor could enhance functional recovery in hemiparkinsonian rats. ${ }^{37}$

PC-12 cells, a monoamine cell line derived from a pheochromocytoma in the adrenal medulla of a male rat, can express tyrosine hydroxylase and synthesize dopamine intracellularly, so are widely used in the study of PD models in vitro. ${ }^{38}$ In our in vitro experiment, we used 6-OHDA to trigger apoptosis and then added SP-GNPs at different concentrations to investigate the effect of SP-GNPs on growth of PC-12 cells. It is evident from the results of these investigations that SP can decrease apoptosis and enhance cell growth to a considerable degree. Further, within a certain range of concentrations, the degree of inhibition of cell apoptosis increases as the concentration of SP increases, with cells growing better and in larger numbers at higher SP concentrations.

In our in vivo experiment, SP-GNPs were administered intranasally to rats with 6-OHDA-induced hemiparkinsonism, and these rats showed more functional improvement and less apoptosis than their counterparts that were not treated with intranasal SP-GNPs. Intranasal administration of SP-GNPs inhibited 6-OHDA-induced apoptosis and improved symptoms of hemiparkinsonism. With increasing concentrations of SP, rats with hemiparkinsonism showed more functional improvement, with further decreases in levels of apoptosis, indicating that the strength of the neuroprotective effect had a positive relationship with the SP concentration in the brain.

As a noninvasive strategy, GNP-mediated intranasal delivery of SP protects against 6-OHDA-induced apoptosis, and might constitute a practical therapy for $\mathrm{PD}$ patients in the future.

\section{Acknowledgments}

This research was supported by grants from the National Natural Science Funds of China (81301982, 81360195, $81272160,81302726,81101570)$, the Major Scientific Project of Guangdong Province (2012A080201010) and the science and technology research of college students in Zhejiang Province (Xinmiao research, 2014R413069).

\section{Disclosure}

The authors report no conflicts of interest in this work.

\section{References}

1. Barbiero JK, Santiago R, Tonin FS, et al. PPAR- $\alpha$ agonist fenofibrate protects against the damaging effects of MPTP in a rat model of Parkinson's disease. Prog Neuropsychopharmacol Biol Psychiatry. 2014;53: 35-44.

2. Fujita H, Ogino T, Kobuchi H, et al. Cell-permeable cAMP analog suppresses 6-hydroxydopamine-induced apoptosis in PC12 cells through the activation of Akt pathway. Brain Res. 2006;1113:10-23.

3. Przedborski S, Ischiropoulos H. Reactive oxygen and nitrogen species: weapons of neuronal destruction in models of Parkinson's disease. Antioxid Redox Signal. 2005;7:685-693.

4. Li X, Ye X, Li X, et al. Salidroside protects against MPP+-induced apoptosis in PC12 cells by inhibiting the NO pathway. Brain Res. 2011; 1382:9-18. 
5. Fiebich BL, Schleicher S, Butcher RD, Craig A, Lieb K. The neuropeptide substance $\mathrm{P}$ activates $\mathrm{p} 38$ mitogen-activated protein kinase resulting in IL-6 expression independently from NF-kappa B. J Immunol. 2000;165:5606-5611.

6. Betaret R, Greenamyre JT. Regulation of dopamine receptor and neuropeptide expression in the basal ganglia of monkeys treated with MPTP. Exp Neurol. 2004;189:393-403.

7. Chen LW, Yung KK, Chan YS. Neurokinin peptides and neurokinin receptors potential therapeutic intervention targets of basal ganglia in the prevention and treatment of Parkinson's disease. Curr Drug Targets. 2004;5:197-206.

8. Thornton E, Tran TT, Vink R. A substance P mediated pathway contributes to 6-hydroxydopamine induced cell death. Neurosci Lett. 2010;481:64-67.

9. Backman LJ, Danielson P. Akt-mediated anti-apoptotic effects of substance $\mathrm{P}$ in Anti-Fas-induced apoptosis of human tenocytes. $J$ Cell Mol Med. 2013;17:723-733.

10. Jiang MH, Lim JE, Chi GF, et al. Substance P reduces apoptotic cell death possibly by modulating the immune response at the early stage after spinal cord injury. Neuroreport. 2013;24:846-851.

11. Krasnova IN, Bychkov ER, Lioudyno VI, Zubareva OE, Dambinova SA. Intracerebroventricular administration of substance P increases dopamine content in the brain of 6-hydroxydopamine-lesioned rats. Neuroscience. 2000;95:113-117.

12. Nikolaus S, Huston JP, Schwarting PK. Pretreatment with fragments of substance $\mathrm{P}$ or with cholecystokinin differential affects recovery from sub-total nigrostriatal 6-hydroxydopamine lesion. Neural Plast. 1999;6:77-89.

13. Thornton E, Vink R. Treatment with a substance P receptor antagonist is neuroprotective in the intrastriatal 6-hydroxydopamine model of early Parkinson's disease. PLoS One. 2012;7:e34138.

14. Thornton E, Ziebell JM, Leonard AV, Vink R. Kinin receptor antagonists as potential neuroprotective agents in central nervous system injury. Molecules. 2010;15:6598-6618.

15. Weinstock JV. Substance $P$ and the regulation of inflammation in infections and inflammatory bowel disease. Acta Physiol (Oxf). 2015;213: 453-461.

16. Lochhead JJ, Thorne RG. Intranasal delivery of biologics to the central nervous system. Adv Drug Deliv Rev. 2012;64:614-628.

17. Sivera M, Kvitek L, Soukupova J, et al. Silver nanoparticles modified by gelatin with extraordinary $\mathrm{pH}$ stability and long-term antibacterial activity. PLoS One. 2014;9:e103675.

18. Elzoghby AO. Gelatin-based nanoparticles as drug and gene delivery systems: reviewing three decades of research. J Control Release. 2013; 172:1075-1091.

19. Lee SJ, Yhee JY, Kim SH, Kwon IC, Kim K. Biocompatible gelatin nanoparticles for tumor-targeted delivery of polymerized siRNA in tumor-bearing mice. J Control Release. 2013;172:358-366.

20. Varshosaz J, Eskandari S, Tabbakhian M. Production and optimization of valproic acid nanostructured lipid carriers by the Taguchi design. Pharm Dev Technol. 2010;15:89-96.

21. Varshosaz J, Tabbakhian M, Mohammadi MY. Formulation and optimization of solid lipid nanoparticles of buspirone $\mathrm{HCl}$ for enhancement of its oral bioavailability. J Liposome Res. 2010;20:286-296.

22. Zare K, Eidi A, Roghani M, Rohani AH. The neuroprotective potential of sinapic acid in the 6-hydroxydopamine-induced hemi-parkinsonian rat. Metab Brain Dis. 2015;30:205-213.
23. Suzuki S, Kawamata J, Iwahara N, et al. Intravenous mesenchymal stem cell administration exhibits therapeutic effects against 6-hydroxydopamine-induced dopaminergic neurodegeneration and glial activation in rats. Neurosci Lett. 2015;584:276-281.

24. Zhang X, Zhu Y, Duan W, Feng C, He X. Allicin induces apoptosis of the MGC-803 human gastric carcinoma cell line through the p38 mitogen-activated protein kinase/caspase-3 signaling pathway. Mol Med Rep. 2015;11:2755-2760.

25. Kabanov AV, Gendelman HE. Nanomedicine in the diagnosis and therapy of neurodegenerative disorders. Prog Polym Sci. 2007;32: 1054-1082.

26. Mistry A, Stolnik S, Illum L. Nanoparticles for direct nose-to-brain delivery of drugs. Int J Pharm. 2009;379:146-157.

27. Vyas TK, Shahiwala A, Marathe S, Misra A. Intranasal drug delivery for brain targeting. Curr Drug Deliv. 2005;2:165-175.

28. Ahmad N, Umar S, Ashafaq M, et al. A comparative study of PNIPAM nanoparticles of curcumin, demethoxycurcumin, and bisdemethoxycurcumin and their effects on oxidative stress markers in experimental stroke. Protoplasma. 2013;250:1327-1338.

29. Pardeshi CV, Belgamwar VS, Tekade AR, Surana SJ. Novel surface modified polymer-lipid hybrid nanoparticles as intranasal carriers for ropinirole hydrochloride: in vitro, ex vivo and in vivo pharmacodynamic evaluation. J Mater Sci Mater Med. 2013;24:2101-2115.

30. Illum $\mathrm{L}$. Transport of drugs from the nasal cavity to the central nervous system. Eur J Pharm Sci. 2000;11:1-18.

31. Thorne RG, Frey WH 2nd. Delivery of neurotrophic factors to the central nervous system: pharmacokinetic considerations. Clin Pharmacokinet. 2001;40:907-946.

32. Dhuria SV, Hanson LR, Frey WH 2nd. Intranasal delivery to the central nervous system: mechanisms and experimental considerations. J Pharm Sci. 2010;99:1654-1673.

33. Costantino HR, Illum L, Brandt G, Johnson PH, Quay SC. Intranasal delivery: physicochemical and therapeutic aspects. Int $J$ Pharm. 2007;337:1-24.

34. Di Colo G, Zambito Y, Zaino C. Polymeric enhancers of mucosal epithelia permeability: synthesis, transepithelial penetration-enhancing properties, mechanism of action, safety issues. J Pharm Sci. 2008;97: 1652-1680.

35. Zaki NM, Awad GA, Mortada ND, Abd ElHady SS. Enhanced bioavailability of metoclopramide $\mathrm{HCl}$ by intranasal administration of a mucoadhesive in situ gel with modulated rheological and mucociliary transport properties. Eur J Pharm Sci. 2007;32:296-307.

36. Vandelli MA, Rivasi F, Guerra P, Forni F, Arletti R. Gelatin microspheres crosslinked with d,1-glyceraldehyde as a potential drug delivery system: preparation, characterization, in vitro and in vivo studies. Int J Pharm. 2001;215:175-184.

37. Zhao YZ, Li X, Lu CT, et al. Gelatin nanostructured lipid carriersmediated intranasal delivery of basic fibroblast growth factor enhances functional recovery in hemiparkinsonian rats. Nanomedicine. 2014;10:755-757.

38. Hu X, Zhang H, Zhang Y, et al. Differential protein profile of PC12 cells exposed to proteasomal inhibitor lactacystin. Neurosci Lett. 2014; 575:25-30.
Drug Design, Development and Therapy

\section{Publish your work in this journal}

Drug Design, Development and Therapy is an international, peerreviewed open-access journal that spans the spectrum of drug design and development through to clinical applications. Clinical outcomes, patient safety, and programs for the development and effective, safe, and sustained use of medicines are a feature of the journal, which

\section{Dovepress}

has also been accepted for indexing on PubMed Central. The manuscript management system is completely online and includes a very quick and fair peer-review system, which is all easy to use. Visit http://www.dovepress.com/testimonials.php to read real quotes from published authors. 